\title{
Viewpoint
}

\section{Switching magnetism with electric fields}

\author{
Wolfgang Kleemann \\ Fachbereich Physik, Universität Duisburg-Essen, 47048 Duisburg, Germany
}

Published December 14, 2009

The magnetic state of a multiferroic device can be controlled purely by an electric field-a property that could greatly reduce overheating in data storage devices.

Subject Areas: Magnetism, Spintronics, Materials Science

\author{
A Viewpoint on: \\ Electric Field Switching of the Magnetic Anisotropy of a Ferromagnetic Layer Exchange Coupled to the Multifer- \\ roic Compound $\mathrm{BiFeO}_{3}$ \\ D. Lebeugle, A. Mougin, M. Viret, D. Colson and L. Ranno \\ Phys. Rev. Lett. 103, 257601 (2009) - Published December 14, 2009
}

Overheating is a major bottleneck in optimizing the performance of microelectronic devices. Following Moore's law, the power density in processors is increasing exponentially and calls for thermal management. With increased integration (current typical length scales being less than $45 \mathrm{~nm}$ base length), 3D packaging, and use of current controlled memory cells (examples of which include nonvolatile MRAM [1] or the "magnetic racetrack" [2]), these issues are coming to a head. Nevertheless, optimistic design engineers promise batteries that last for 24 hours in the next generation of laptop computers, with minimal heat dissipation at highest performance. What strategies do they have in mind?

One promising route to this goal is purely electricfield-controlled devices, which cause much less Joule heating than magnetic devices that are switched with a current. This technological need revived interest in magnetoelectric (ME) materials [3], in which magnetism can be controlled via electric fields, and vice versa [4]. Its promise reaches its zenith in multiferroics. These materials that undergo phase transitions into long range magnetic and electric order are potentially the most promising materials for electric control of magnetism, for storage and for processing. Figure 1 shows a visionary sketch of a voltage-controlled write head at a hard disk track [5], where the magnetic "write" field is generated by an electric field in a magnetoelectric material via its bulk magnetic moment, $\Delta m_{\mathrm{ME}}$, and its magnetic flux guided to a writing gap.

Unfortunately, the linear magnetoelectric effect is so small in most materials [3] that this direct approach has no chance of being successful in practice. However, much effort has been put into related devices. Binek and Doudin [6] were probably the first to draw attention to the potential of spintronic concepts in this pursuit, when they proposed to use magnetoelectric materials as active tunnel barriers or pinning layers of spin-dependent

DOI: $10.1103 /$ Physics.2.105

URL: http://link .aps.org/doi/10.1103/Physics.2.105 transport devices like magnetic random access memory (MRAM) [1]. In contrast to Fig.1, $\Delta m_{\mathrm{ME}}$ now controls the magnetization state of adjacent ferromagnetic (FM) layers via exchange coupling, which is the crucial keyword in all actual device proposals. It is this that has motivated a paper published in Physical Review Letters by Lebeugle et al.[7], who have demonstrated electric field switching of the magnetic anisotropy of a soft magnetic layer of $\mathrm{Ni}_{0.78} \mathrm{Fe}_{0.22}$ ("NiFe" or Permalloy) attached to a single crystal of ferroelectric and antiferromagnetic $\mathrm{BiFeO}_{3}$ (BFO).

The results by Lebeugle et al. should be regarded in parallel to very similar experiments of Ramesh's group [8] on soft magnetic layers of $\mathrm{Co}_{0.9} \mathrm{Fe}_{0.1}$ ("CoFe") attached to thin films of BFO. Both composites involve the canonical multiferroic BFO [9], still the only roomtemperature multiferroic with exceptionally high ordering temperatures: ferroelectric Curie temperature $T_{C}=$ $1103 \mathrm{~K}$ and antiferromagnetic Néel temperature $T_{N}=$ $643 \mathrm{~K}$

In both Refs. [7] and [8], it has been shown that an electric field-induced change of the ferroelectric polarization of the BFO substrate is able to toggle the easy direction of the magnetization in the ferromagnetic layer by use of the magnetoelectric effect. More precisely, two successive coupling mechanisms are exploited. The first is the magnetoelectric coupling within BFO between the antiferromagnetic and the ferroelectric order. The mechanisms were described by the same groups [7, 8] either as an electric field-induced spin reorientation ("spin flop") [10] or as an indirect magnetoelectric effect mediated by the ferroelastic stress accompanying the ferroelectric domains [11]. As a matter of fact, it is found that the ferroelectric domains, that is, regions with different collective polarization, go perfectly with the antiferromagnetic ones due to the accompanying lattice strain.

The second coupling process is based on exchange (C) 2009 American Physical Society 


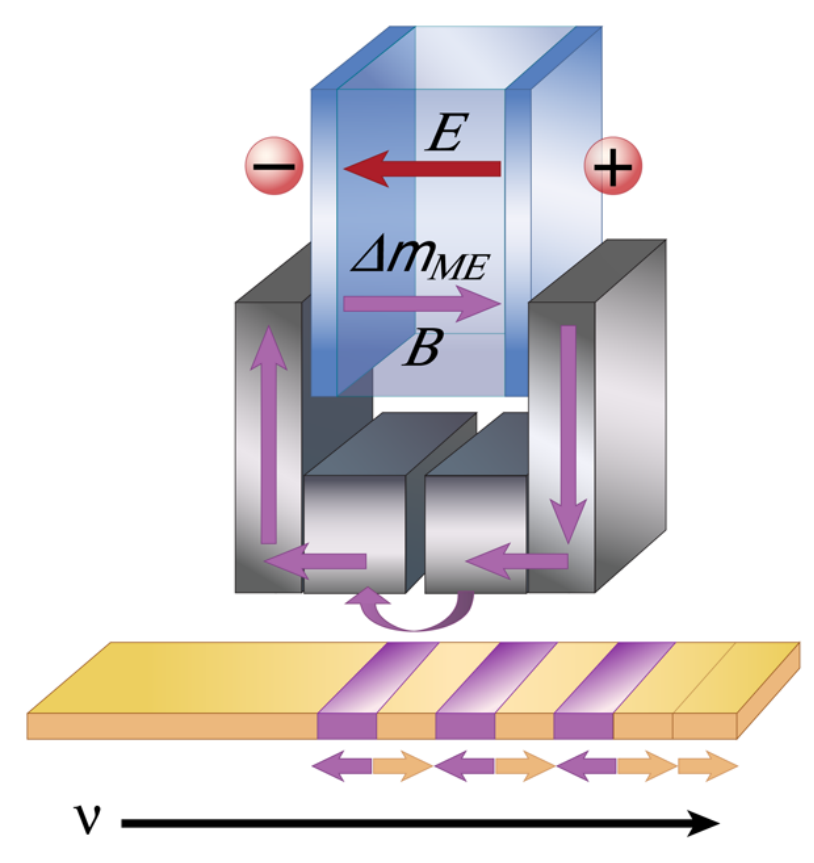

FIG. 1: Sketch of a magnetoelectric write head for magnetic hard disk (after A. K. Zvezdin [5]). An electric field E generates a bulk magnetic moment $\Delta m_{\mathrm{ME}}$ in a slab of magnetoelectric material, thus giving rise to a magnetic flux density $\mathbf{B}$. The gap in the flux closing yoke emits a stray field, which writes a magnetic "bit" into the moving track (velocity $v$ ) of the hard disk. (Illustration: Carin Cain)

interactions at the interface between the antiferromagnetic BFO and the ferromagnetic Permalloy (or CoFe). More precisely, it is the projection of the antiferromagnetic order that couples to the ferromagnetic magnetization. Slightly different interpretations are in order, since the $\mathrm{Fe}^{3+}$ spins of BFO form cycloids in the single crystal [7], while they are uniformly canted in the thin film [8]. The cycloidal propagation vector favors a parallel alignment with the Permalloy spins. As a result the effective coupling is several times larger in the crystal than in the film. Unfortunately, the magnetization of the Permalloy layer could not be completely switched along the directions of the anisotropy axes, as it is impossible to form a ferroelectric single domain in the (001) plane of BFO. Out of the manifold of eight differently poled domains, only four of them can be selected by a uniform intraplanar field. Similar results emerged from the switching experiments on $\mathrm{BFO} / \mathrm{CoFe}$ bilayers [8].

At this point one might ask whether BFO really is the most promising material for switching magnetism with an electric field. The remaining ambiguity of electric switching due to crystallographic multiaxiality obviously calls for a uniaxial magnetoelectric material exhibiting only two different $180^{\circ}$ ferroelectric, ferromagnetic, or antiferromagnetic domains. We found that the primordial magnetoelectric material, chromium oxide $\mathrm{Cr}_{2} \mathrm{O}_{3}$ [12], fulfils this requirement in an unprecedented way. Its two uniaxial antiferromagnetic domains can be switched magnetoelectrically, by simultaneously applying a magnetic and an electric field, $\mathbf{E}$ and $\mathbf{H}$, on cooling to below $T_{N}=308 \mathrm{~K}[13]$ or isothermally in a large enough field product $\mathbf{E} \cdot \mathbf{H}[14]$. By keeping $\mathbf{H}$ constant (e.g., with a permanent magnet) the antiferromagnetic single domain state can be switched with the electric field $\pm \mathbf{E}$. This has spectacular consequences to an attached ferromagnetic layer, whose magnetization is also switched by $180^{\circ}$ due to the so-called exchange bias effect [15]. We showed this on a ferromagnetic trilayer $\mathrm{Pt} / \mathrm{Co} / \mathrm{Pt}$ on top of a (0001) oriented single crystal of $\mathrm{Cr}_{2} \mathrm{O}_{3}$ [16]. Following earlier ideas [6], the design of magnetoelectric random access memory and logic cells, MERAM and MEXOR [17], was obvious. Their binary information storage is based on the low and high tunnel magnetoresistances of the magnetoelectric switchable "free layer" coupled by a tunnel barrier to a "pinned layer" with fixed ferromagnetic magnetization, parallel or antiparallel to the "free" one, respectively. The next steps to take toward possible applications will be the reduction of leakage currents in $\mathrm{Cr}_{2} \mathrm{O}_{3}$ thin films and the increase of their Néel temperature, e.g., by alloying with $\mathrm{Fe}_{2} \mathrm{O}_{3}$.

In conclusion, the present strategies of switching magnetism with electric fields-targeting either ferromagnetic anisotropy or exchange bias-while still in their infancy, are highly promising, though both fundamental and material issues still have to be solved.

\section{References}

[1] J. Åkerman, Science 308, 508 (2005).

[2] S. P. S. Parkin, M. Hayashi, and L. Thomas, Science 320, 190 (2008).

[3] M. Fiebig, J. Phys. D 38, R123 (2005).

[4] L. D. Landau and E. M. Lifshitz, Electrodynamics of Continuous Media (Pergamon Press, Oxford, 1975).

[5] A. K. Zvezdin, "Nanomagnetism and spintronics," Lille, 2005 (unpublished).

[6] Ch. Binek and B. Doudin, J. Phys. Condens. Matter 17, L39 (2005).

[7] D. Lebeugle, A. Mougin, M. Viret, D. Colson, and L. Ranno, Phys. Rev. Lett. 103, 257601 (2009).

[8] Y.-H. Chu, L. W. Martin, M. B. Holcomb, M. Gajek, S.-J. Han, Q. He, N. Balke, C.-H. Yang., D. Lee, W. Hu, Q. Zhan, P.-L. Yang, A. Fraile-Rodriguez, A. Scholl, S. X. Wang, and R. Ramesh, Nature Mater. 7, 478 (2008).

[9] A. M. Kadomtseva, Yu. F. Popov, A. P. Pyatakov, G. P. Vorob'ev, A. K. Zvezdin, and D. Viehland, Phase Trans. 79, 1019 (2006).

[10] D. Lebeugle, D. Colson, A. Forget, M. Viret, A. M. Bataille, and A. Gukasov, Phys. Rev. Lett. 100, 227602 (2008).

[11] T. Zhao, A. Scholl, F. Zavaliche, K. Lee, M. Barry, A. Doran, M. P. Cruz, Y. H. Chu, C. Ederer, N. A. Spaldin, R. R. Das, D. M. Kim, S. H. Baek, C. B. Eom, and R. Ramesh, Nature Mater. 5, 823 (2006).

[12] D. N. Astrov, Sov. Phys. JETP 11, 708 (1960).

[13] T. H. O'Dell, Electrodynamics of Magneto-Electric Media (NorthHolland, Amsterdam, 1970).

[14] T. J. Martin and J. C. Anderson, IEEE Trans. Magn. 2, 446 (1966).

[15] W. H. Meiklejohn and C. P. Bean, Phys. Rev. 102, 1413 (1956).

[16] P. Borisov, A. Hochstrat, X. Chen, W. Kleemann, and Ch. Binek, Phys. Rev. Lett. 94, 117203 (2005).

[17] X. Chen, A. Hochstrat, P. Borisov, and W. Kleemann, Appl. Phys. Lett. 89, 202508 (2006); U.S. Patent No. 11/909,854 (pend.). 


\section{About the Author}

\section{Wolfgang Kleemann}

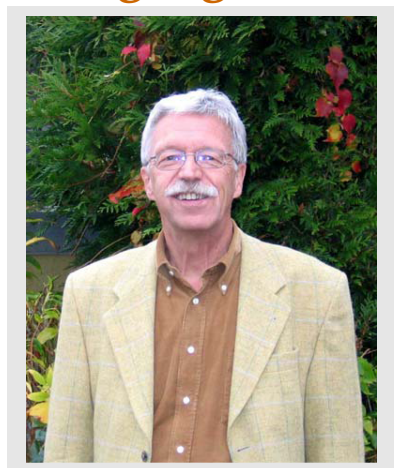

Wolfgang Kleemann is professor of experimental and applied physics at the University of Duisburg-Essen. He received his Ph.D. in 1968 from the University of Göttingen and became professor at the Universities of Paderborn (1975) and Duisburg (since 1982). His perpetual research interest in both magnetism (ferro- and antiferromagnetic crystals, multilayers and nanoparticles) and ferroelectricity (crystals, films and ceramics) brought him to Universities like Paris-Sud, UC Santa Barbara, RIKEN Tokyo, SNU Seoul, and UFMG Belo Horizonte as a visiting professor. His activities have recently become focused on magnetoelectric and multiferroic materials. 